Background: Osteoporosis is an increasingly important health problem among patients with spondyloarthritis (SPA). The Measure of Bone Mineral Density BMD is routinely carried out in an anteroposterior (AP) view of the spine. However, the syndesmophytes, ligaments calcifications, and the posterior part of vertebrae affect AP scanning. A lateral spine view is a more sensitive tool in assessing bone loss in trabecular bone.

Objectives: We aimed to evaluate the association between lateral lumbar DXA and syndesmophyte grading in patients with SPA.

Methods: We conducted a retrospective study including 75 patients with SPA. Bone density of the hip and lumbar spine was measured with a GE Lunar Prodigy Advance Bone Densitometer equipment. All patients had lumbar lateral, AP, and proximal femur DXA scans. The T-score, which measures the difference between a patient's BMD and young-normal subjects, was computed and age-matched. Results: The mean age of the patients was $36 \pm 11$ years. Male predominance was noted with a sex ratio of 4.76 . The mean BMI was $25 \pm 5 \mathrm{~kg} / \mathrm{m} 2$. Eight percent were obese. Fifty-two percent had Vitamin D deficiency.

Forty-eight percent of the patients had axial SPA, while $52 \%$ had axial and peripheral symptoms.

The mean age of onset was $27 \pm 7$ years. Fifty-two percent of the patients had high inflammatory biomarkers. The BASDAI, ASDAS-VS, and ASDAS-CRP mean levels were respectively: $3.5 \pm 2.4,3.1 \pm 0.9$, and $3 \pm 0.8$. The mean $B A S R I$ and mass were respectively $8+4.8$ and $16.4+19.4$. Analyses of T-score values obtained over the femoral neck revealed osteoporosis in $18.7 \%$ of the cases and osteopenia in $32 \%$ of the cases. On the other hand, analyses of AP, spine views revealed osteoporosis in $25.3 \%$ and osteopenia in $45.3 \%$ of patients ( $p=0.028, r=0.254)$. We detected the highest percentage of osteoporosis in lateral lumbar view and T-scores matched more closely with femoral neck values; osteoporosis in $29.3 \%$, and osteopenia in $22.7 \%$ of the patients $(p<10-3, r=0.562)$. BMD measured in AP, and lateral views were in good agreement $(p<10-3, p=0.592)$. Age was inversely but not significantly associated with BMD in lateral $(p=0.442, r=-0.09)$, AP $(p=0.319, r=-0.117)$ and femoral neck projections $(p=0.179, r=-0.157)$. Femoral neck BMD was associated with the activity of SPA (ASDAS vs $(p=0.027, r=-0.295)$, and the mobility limitation BASMI ( $p=0.032, r=-0.247)$. Coxitis, BASRI, or mSASS were independent of BMD. Conclusion: We conclude that spine lateral view in DXA accurately measures BMD exceeding the AP spine views and femoral neck values. Therefore, structural changes do not affect this measurement

Disclosure of Interests: None declared.

DOI: 10.1136/annrheumdis-2021-eular.4294

\section{POS1019 INCONSISTENCY OF THE DEGREE OF CARDIOVASCULAR RISK WHEN ASSESSED USING DIFFERENT INDICES IN PATIENTS WITH AXIAL SPONDYLOARTHRITIS}

E. Vasilenko ${ }^{1}$, A. Dadalova ${ }^{1}$, R. Samigullina ${ }^{1}$, V. Mazurov ${ }^{1} .{ }^{1}$ North-Western State Medical University named after I. I. Mechnikov, St. Petersburg, Russia, Department of Therapy, Rheumatology, Examination of Temporary Disability and Quality of Medical Care named after E.E. Eichwald, St. Petersburg, Russian Federation

Background: Evaluation of indicators of cardiovascular risk is one of the main tasks facing a rheumatologist in the tactics of choosing a therapy for patients (pts) with axial spondyloarthritis (axSpA). It is known that pts suffering from axSpA are characterized by a significant increase in cardiovascular risk (CVR). However, there are still no recommendations regulating risk assessment scales in pts with axSpA.

Objectives: were to assess the CVR in pts with axSpA and to compare different cardiovascular risk scales in these pts.

Methods: The study included 55 pts at the age of 45-65 years with diagnosis of axSpA fulfilling ASAS criteria (2009) from St. Petersburg' axSpA register. Three indices of cardiovascular risk evaluation (Systematic COronary Risk Evaluation (SCORE) with increasing coefficient 1.5 for inflammatory diseases, Reynolds Risk Score (RRS), and the third modification of QRESEARCH Cardiovascular Risk Algorithm (QRISK3) were calculated. Risk gradation: low risk $(<1 \%)$, medium (1.0-4.9\%), high (5.0-9.9\%), very high (> 10\%).

Results: Mean age of the pts was $45.8 \pm 10.3$ years; males - $37(67.3 \%)$ pts, HLAB27 positive $-34(61.8 \%)$; mean disease duration $12.5 \pm 8.7$ years. Mean value of SCORE was $2.83 \pm 1.89 \%$, of RRS $-5.04 \pm 3.98 \%$, of QRISK3 $-7.91 \pm 4.91 \%$.

The gradation of the degree of risk depending on the applied assessment index is presented in Table 1 .

\begin{tabular}{lccc}
\hline Index & \multicolumn{3}{c}{ Results } \\
\hline Risk degree & SCORE & RRS & QRISK3 \\
\hline Low & $21(38,2 \%)$ & $8(14,5 \%)$ & $0(0,0 \%)$ \\
Medium & $26(47,3 \%)$ & $23(41,8 \%)$ & $17(30,9 \%)$ \\
High & $8(14,5 \%)$ & $17(30,9 \%)$ & $22(40,0 \%)$ \\
Very high & $0(0,0 \%)$ & $7(12,7 \%)$ & $16(29,1 \%)$
\end{tabular}

Particular attention is drawn to the $100 \%$ discrepancy of low risk values when comparing SCORE and QRISK3. A similar trend persisted when comparing medium, high and very high risk. Thus, the assessment of the risks of 10 -year significant cardiovascular events in pts with axSpA using the SCORE index does not coincide with the QRISK3 index data in $87.27 \%$ of cases, and with the RRS data - in $58.18 \%$ of cases. In $84.3 \%$ of cases, the mismatch between the SCORE and RRS indexes was due to the presence of an increased CRP level.

Conclusion: When assessing cardiovascular risk in pts with axial spondyloarthritis, a discrepancy was found between the degrees of risk when assessed using different scales. SCORE scores were significantly different from Reynolds and QRISK3 scores. These features can be interrelated with a small number of factors assessed when calculating the SCORE, even though there is a correction factor for rheumatic diseases. For pts with axial spondyloarthritis, it is necessary to use additional indicators that influence cardiovascular risk, such as CRP. Disclosure of Interests: None declared. DOI: 10.1136/annrheumdis-2021-eular.4311

\section{Psoriatic arthritis - treatment}

\begin{tabular}{|l|l}
\hline POS1020 & EFFICACY OF TOFACITINIB ON DACTYLITIS IN \\
INDIVIDUAL DIGITS IN PATIENTS WITH ACTIVE \\
PSORIATIC ARTHRITIS
\end{tabular}

A. M. Orbai ${ }^{1}$, P. J. Mease ${ }^{2,3}$, P. Helliwell ${ }^{4}$, O. Fitzgerald ${ }^{5}$, M. Bdewi ${ }^{6}$, D. Fleishaker ${ }^{7}$, R. Mundayat ${ }^{8}$, P. Young ${ }^{9} .{ }^{1}$ Johns Hopkins University School of Medicine, Division of Rheumatology, Baltimore, MD, United States of America; ${ }^{2}$ Swedish Medical Center/Providence St Joseph Health, Rheumatology Clinical Research Division, Seattle, WA, United States of America; ${ }^{3}$ University of Washington School of Medicine, Rheumatology Clinical Research Division, Seattle, WA, United States of America; ${ }^{4}$ Leeds Institute of Rheumatic and Musculoskeletal Medicine, University of Leeds, Leeds, United Kingdom; ${ }^{5}$ Conway Institute for Biomolecular Research, University College Dublin, Dublin, Ireland; ${ }^{6}$ College of Medicine, King Saud University Medical City, King Saud University, Division of Rheumatology, Riyadh, Saudi Arabia; ${ }^{7}$ Pfizer Inc, Inflammation and Immunology, Groton, CT, United States of America; ${ }^{8}$ Pfizer Inc, Inflammation and Immunology, New York, NY, United States of America; ${ }^{9} \mathrm{P}$ fize Inc, Inflammation and Immunology, Collegeville, PA, United States of America

Background: Dactylitis, a hallmark of psoriatic arthritis (PsA), is a uniformly diffuse and sometimes painful swelling of the fingers and/or toes. ${ }^{1}$ Up to $50 \%$ of patients (pts) with PsA may experience dactylitis; ${ }^{1,2}$ as such, dactylitis is an accepted domain of PsA that should be considered in treatment decisions. ${ }^{3}$ In PsA, dactylitis typically involves feet more than hands; dactylitic joints more frequently have erosive damage, compared with non-dactylitic joints. ${ }^{2}$ There remains a need for effective therapies to treat dactylitis in pts with PsA. Improvements in dactylitis have been associated with tofacitinib, an oral Janus kinase inhibitor for the treatment of PsA. ${ }^{4,5}$

Objectives: To assess the effect of tofacitinib on dactylitis by location (hands/ feet) and individual digit involvement in pts with PsA.

Methods: These post hoc analyses used data pooled from two Phase 3 studies (12-month OPAL Broaden [NCT01877668]; 6-month OPAL Beyond $\left[\mathrm{NCT}_{01882439}\right]^{4}$ ) in pts with active PsA treated with tofacitinib $5 \mathrm{mg}$ twice daily (BID; approved dose; to Month [M] 6), tofacitinib $10 \mathrm{mg}$ BID (to M6) or placebo (PBO; to M3); pts were treated continuously with a single conventional synthetic disease-modifying antirheumatic drug. Pts were categorised by the presence of dactylitis at baseline $(B L)$ in the hands and/or feet. Endpoints included change from BL in Dactylitis Severity Score (DSS), ${ }^{6}$ the number of dactylitic digits and the proportion of pts with dactylitis in individual digits at M1, M3 and M6. Descriptive statistics were generated by visit and treatment arm.

Results: Data were pooled from 373 pts with DSS $>0$ at BL. BL characteristics including gender, age, race, body mass index, PsA duration, BL DSS and dactylitic digits count were similar across dactylitis groups and treatment groups, except for pts with dactylitis in both the hands and feet, who had higher DSS compared to those with dactylitis in the hands or feet only, likely due to having more dactylitic digits (data not shown). Regardless of location, pts treated with tofacitinib had cumulative improvements from BL to M6 in DSS (Figure 1a) and in the number of dactylitic digits (Figure 1b); improvements in DSS were greater at M1 and M3, compared with PBO. Pts treated with tofacitinib $10 \mathrm{mg}$ BID typically had numerically greater improvements in DSS, compared with pts treated with tofacitinib $5 \mathrm{mg}$ BID (Figure 1a). Most pts treated with tofacitinib experienced improvement of dactylitis across all fingers and toes (Figure 1c-f); mean dactylitis presence was $\leq 15 \%$ at M 6 in pts treated with tofacitinib for all digits. Generally, at M1 and M3, fewer pts treated with tofacitinib had dactylitis in any digit, compared with PBO (Figure 1c-f).

Conclusion: Among pts with pre-existing dactylitis, treatment with tofacitinib resulted in improvements in dactylitis in hands, feet, or both, and in all digits as early as M1, and up to M6. 